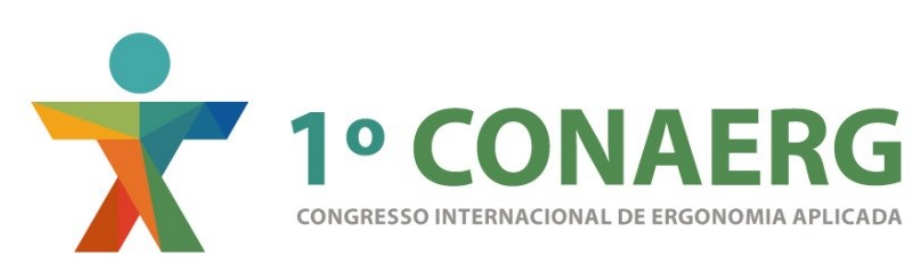

\title{
MÉTODOS NEUROERGONÔMICOS DE MENSURAÇÃO DA CARGA MENTAL DE TRABALHO
}

\author{
Ravenna Leite da Silva (1) \\ Luiz Bueno da Silva (2) \\ Ricardo Moreira da Silva (3) \\ (1) Universidade Federal da Paraíba/Programa de Pós Graduação em Engenharia de \\ Produção, Especialista/Mestranda. \\ E-mail: ravennaleite3@gmail.com \\ (2) Universidade Federal da Paraíba/Programa de Pós Graduação em Engenharia de \\ Produção, Pós-Doutor. \\ E-mail: bueno@ct.ufpb.br \\ (3) Universidade Federal da Paraíba/Programa de Pós Graduação em Engenharia de \\ Produção, Pós-Doutor. \\ E-mail: ricardomoreira0203@hotmail.com

\begin{abstract}
RESUMO
Neuroergonomia é definida como o estudo da atividade do cérebro humano em relação ao trabalho e integra abordagens de ergonomia e neurociência. Este estudo trata-se de uma revisão de literatura realizada nas bases de dados Science Direct, Scopus, Pubmed e Web of Science usando as palavras-chaves: neuroergonomics e mental workload. Foi realizado um levantamento inicial sobre métodos neuroergonômicos de mensuração da carga de trabalho mental. A neuroergonomia tem contribuído para a sua avaliação e opera como uma ferramenta adicional tanto em teorias de refino da mesma, como no fornecimento de técnicas alternativas para sua avaliação em ambientes de trabalho.
\end{abstract}

Palavras-chaves: Neuroergonomia; Carga de Trabalho Mental; Métodos de avaliação.

\begin{abstract}
Neuroergonomics is defined as the study of the activity of the human brain in relation to work and integrate ergonomics and neuroscience approaches. This study deals with a literature review conducted in Science Direct, Scopus, Pubmed and Web of Science databases using the keywords: neuroergonomics and mental workload. An initial survey neuroergonomics methods for measurement of mental workload was performed. The neuroergonomics has contributed to the review and operates as an additional tool in both refining theories of it, as in the provision of alternative techniques for evaluation in work environments.
\end{abstract}


Keywords: Neuroergonomics; Mental Workload; Evaluation methods.

\section{INTRODUÇÃO}

Segundo Másculo e Vidal (2011) ergonomia é uma ocupação de pessoas qualificadas para responder às demandas quanto à atividade de trabalho. Essas questões estabelecem campos de interesse amplo e diversificado que vão desde temas sobre anatomia à teoria das organizações, do cognitivo ao social, do conforto à prevenção de acidentes. Já de acordo com a International Ergonomics Association (IEA), é a disciplina científica que trata da compreensão das relações entre os seres humanos e outros elementos de um sistema, e a profissão que aplica teorias, princípios, dados e métodos, a projetos que visam otimizar o bem estar humano e a performance global dos sistemas (INTERNATIONAL ERGONOMICS ASSOCIATION, 2008).

Ainda utilizando as definições da IEA, a ergonomia pode ser classificada em: física, cognitiva e organizacional. Para fins didáticos e melhor compreensão deste trabalho faz-se necessário a compreensão da ergonomia cognitiva que abrange os processos mentais, tais como percepção, memória, raciocínio e resposta motora, conforme afetam interações entre seres humanos e outros elementos de um sistema. Os tópicos relevantes incluem carga mental de trabalho, tomada de decisão, performance especializada, interação homemcomputador, stress e treinamento conforme estes se relacionam aos projetos envolvendo seres humanos e sistemas (INTERNATIONAL ERGONOMICS ASSOCIATION, 2008).

Sendo um adendo da ergonomia, a neurociência segundo Uehara e Morais (2011) revela a existência de redes neurais, que formam diversos sistemas cerebrais específicos na execução de determinada função cognitiva. A partir disso, pode-se observar com mais precisão, por exemplo, o tempo e as estruturas específicas envolvidas na execução de determinada tarefa ou na realização de operações mentais particulares. Ao se aplicar tais possibilidades de estudo nos trabalhos de ergonomia, podemos notar uma mudança na abordagem adotada. Assim, a neuroergonomia é definida como o estudo do cérebro humano em relação ao desempenho em ambientes de trabalho e seus ajustes diários. Integra teorias e princípios de ergonomia, neurociência e fatores humanos para fornecer informações valiosas sobre o funcionamento do cérebro e comportamento como encontrado em ambientes naturais (METHA;PARASURAMAN, 2013).

Para os citados autores, os estudos na neuroergonomia dependem fortemente das técnicas de neuroimagem para compreender as estruturas cerebrais, os mecanismos e funções durante o trabalho. Essas técnicas dividem-se em duas categorias gerais: (i) aquelas que são indicadores diretos da atividade neuronal em resposta a estímulos, tais como Eletroencefalografia (EEG) e Potenciais Relacionados com Eventos (ERPs), e (ii) aqueles que fornecem indicadores metabólicos indiretos de atividade neuronal, tais como Ressonância Magnética Funcional (fMRI), Tomografia por Emissão De Pósitrons (PET), e Espectroscopia Funcional no Infravermelho Próximo (fNIRS).

Métodos de neuroimagem têm vindo a melhorar em sua capacidade técnica, sofisticação e amplitude de aplicação ao longo da última década. Estudos de neurociência cognitiva usando esses métodos também têm aumentado em sua complexidade, e tem havido crescente interesse no seu uso para examinar os circuitos neurais que suportam tarefas complexas representante da percepção, cognição e ação à medida que ocorrem em ambientes naturais (PARASURAMAN, 2012) e quando ocorrem no trabalho, nesse artigo, chamamos de carga mental de trabalho.

A carga mental de trabalho é entendida como a interação entre as exigências da tarefa e a capacidade humana de realização. A mesma integra aspectos psíquicos e cognitivos abrangendo os conceitos da carga psíquica (se relacionam aos aspectos afetivos/significação do trabalho) e cognitivos (refere-se às cargas provenientes das exigências cognitivas da tarefa) ao mesmo tempo (CARDOSO; GONTIJO, 2012). 
Para Motter, Santos e Guimarães (2015) o conceito de carga de trabalho é derivado dos estudos da psicologia do trabalho e da ergonomia da atividade. O mesmo tem sido importante para esclarecer questões relacionadas à saúde física e mental do trabalhador. Antonelli (2011) complementa este conceito afirmando que "carga de trabalho é uma medida quantitativa e/ou qualitativa do nível de atividade motora, fisiológica e mental necessária à realização de um trabalho".

Benedetto et al. (2011) conceitua como a quantidade de trabalho mental ou esforço que um indivíduo faz para executar uma tarefa. Kramer e Parasuraman (2007) afirmam que a avaliação da carga mental de trabalho continua a ser um importante tema na pesquisa e prática em ergonomia. De uma perspectiva neuroergonômica, carga mental pode ser considerada como um conjunto composto de cérebro/mente que modula o desempenho humano de perceptual, cognitivas e tarefas motoras.

Diante das definições acima realizadas, é importante entendemos a abordagem neuroergonômica a respeito da carga de trabalho mental. Para tanto é necessário o conhecimento sobre as técnicas utilizadas para análise da carga de trabalho mental, assim como, as características, vantagens e desvantagens de cada uma. Nesse sentido, podemos observar a carência de estudos nacionais em relação ao tema e faz-se necessário que pesquisa brasileira volte-se também para esta área. Portanto, o presente estudo objetiva realizar um breve estado da arte sobre a neuroergonomia na avaliação da carga de trabalho mental.

\section{MATERIAIS E MÉTODOS}

Foi realizada uma revisão de literatura com pesquisa nas bases de dados: Science Direct, Pubmed e Scopus, usando o seguinte algoritmo para busca neuroergonomics and mental workload. Entre os anos 2011 e 2016 foi aplicado o seguinte filtro nas buscas de artigos que contivessem essas palavras no título, resumo e/ou nas palavras-chaves, onde se totalizou apenas 32 referências. Em seguida foram avaliados e retirados os artigos que estivessem repetidos. Após essa última etapa foram selecionados 16 artigos (Gráfico 1).

Gráfico 1. Número de artigos finais selecionados

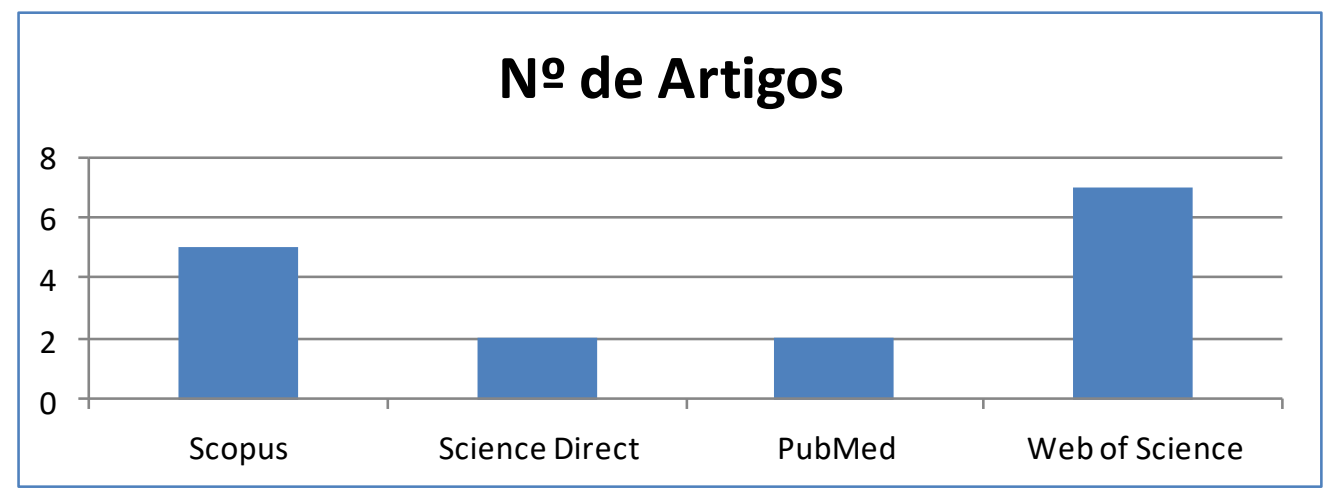

Fonte: Elaborado pelos autores

\section{MÉtOdOS NEUROERGONÔMICOS DE AVALIAÇÃO DA CARGA DE TRABALHO MENTAL}

Os métodos que serão avaliados estão apresentados no Quadro 1 a seguir:

Quadro 1. Lista das técnicas neuroergonômicas e o atributo medido

\begin{tabular}{|l|l|}
\hline Método & Mensura/Verifica \\
\hline MRI (Magnetic Resonance Image)- Ressonância Magnérica & Volume da massa cinza \\
\hline DTI - (Diffusion Tensor Imaging)Tensor de Difusão de Imagem & $\begin{array}{l}\text { Difusão de moléculas de } \\
\text { água nos axónios que }\end{array}$ \\
\hline
\end{tabular}




\begin{tabular}{|c|c|}
\hline & ostäncia \\
\hline $\begin{array}{ll}\text { fMRI (Funcional Magnetic Resonance Imaging) } & \text { Ressonância } \\
\text { Magnética Funcional e PET- (Positron Emission Tomography) } \\
\text { Tomografia por Emissão de Pósitrons }\end{array}$ & Hemodinâmica cerebral \\
\hline $\begin{array}{l}\text { fNIRS - (Functional Near Infrared Spectroscopy) } \\
\text { Espectroscopia Funcional no Infravermelho Próximo }\end{array}$ & $\begin{array}{l}\text { Oxihemoglobina } \\
\text { Deoxihemoglobina }\end{array}$ \\
\hline $\begin{array}{l}\text { TCDS - (Transcranial Doppler Sonography)- Dopler } \\
\text { Transcraniano }\end{array}$ & $\begin{array}{l}\text { Flutuações } \text { da } \\
\text { velocidade de fluxo } \\
\text { sanguíneo cerebral }\end{array}$ \\
\hline EEG - (Electroencephalography) -Eletroencefalograma & $\begin{array}{l}\text { Atividade elétrica pós- } \\
\text { sináptica }\end{array}$ \\
\hline $\begin{array}{l}\text { ERP - (Event-related Potentials) - Potenciais Relacionados a } \\
\text { Eventos }\end{array}$ & $\begin{array}{l}\text { Resposta neural do } \\
\text { cérebro para eventos } \\
\text { específicos. }\end{array}$ \\
\hline $\begin{array}{l}\text { TMS - (Transcranial Magnetic Stim } \\
\text { Magnética Transcraniana e tDCS(Tra } \\
\text { Stimulation) - Estimulação Elétrica Tran }\end{array}$ & $\begin{array}{l}\text { Inibição temporária ou } \\
\text { ativação de regiões } \\
\text { cerebrais específicas. }\end{array}$ \\
\hline
\end{tabular}

Fonte: Dos autores a partir de Metha e Parasuraman (2013).

\subsection{Ressonância Magnética (Magnetic Resonance Image - MRI)}

A técnica em questão fornece uma imagem estrutural do cérebro e oferece excelente visualização espacial das partes internas profundas, tais como o hipocampo. Enquanto ressonância magnética fornece imagens estáticas do cérebro que é fundamental para examinar as mudanças estruturais no cérebro devido a doenças (como tumor), a sua aplicação no estudo de mudanças estruturais no cérebro ao longo do tempo (ou seja, a plasticidade) forneceu informações importantes sobre aprendizagem e formação (METHA;PARASURAMAN, 2013).

Normalmente fisicamente o equipamento é grande, implicando na não mobilidade e no alto custo de adquisição e manutenção.

\subsection{Tensor de Difusão de Imagem (Diffusion Tensor Imaging - DTI)}

Trata-se de uma técnica relativamente nova de ressonância magnética. A mesma utiliza a ressonância magnética para segmentar a difusão de moléculas de água nos axónios que compõem a substância branca do cérebro e permite o cálculo de anisotropia fraccionada (AF).

Os valores de AF podem variar de 0 a 1, em que 0 indica não direccional (isotrópico) e 1 indica perfeitamente direccional (anisotrópica) difusão. Maiores valores de AF são pensados para refletir uma maior integridade da substância branca ligando diferentes regiões corticais e subcorticais do cérebro.

O equipamento também apresenta-se fisicamente volumoso, implicando na não mobilidade e no alto custo de adquisição e manutenção. Vários estudos recentes têm avaliado a eficácia do treinamento cognitivo e motor da integridade da substância branca usando a técnica de DTI (METHA;PARASURAMAN, 2013).

3.3 Ressonância Magnética Funcional (Funcional Magnetic Resonance Imaging - fMRI) e Tomografia por Emissão de Pósitrons (Positron Emission Tomography - PET)

Técnicas que avaliam a hemodinâmica cerebral, como a fMRI e PET fornecem informações valiosas sobre locais de origem dos padrões de ativação neural distintos associados com funções cognitivas, motoras e afetivas simples e complexas.

Enquanto a PET utiliza marcadores radioativos injetados para medir o fluxo sanguíneo em resposta a estímulos, baseada nas respectivas características magnéticas a fMRI incide 
sobre o contraste resultante entre o sangue oxigenado e desoxigenado chamado de Sinal Dependente do Nível de Oxigenação no Sangue ou sinal BOLD (em inglês, Blood Oxygenation Level Dependent) (POLDRACK; et al. 2011).

Tanto fMRI e PET têm sido fundamentais no avanço dos nossos conhecimentos sobre as funções e mecanismos cerebrais durante tarefas motoras e cognitivas. No entanto, esses métodos apresentam algumas limitações, como, por exemplo, a baixa mobilidade e altos custos. Existe uma crescente necessidade de examinar os padrões de ativação cerebral em tarefas complexas em situações cotidianas naturais, que são mais representativas da atividade, e isso tem levado pesquisadores a adotar técnicas de neuroimagem que oferecem melhores características de mobilidade (METHA; PARASURAMAN, 2013).

\subsection{Espectroscopia Funcional no Infravermelho Próximo (Functional Near Infrared} Spectroscopy - fNIRS)

Espectroscopia Funcional no Infravermelho Próximo é uma técnica não-invasiva utilizada para medir a hemodinâmica cerebral semelhante ao PET e fMRI mas com menor resolução espacial. Foram encontrados os seguintes trabalhos:

Quadro 2. Artigos selecionados- fNIRS.

\begin{tabular}{|c|c|c|c|}
\hline Autores & Ano/Base & Título & Palavras-chaves \\
\hline $\begin{array}{l}\text { Carrieri et } \\
\text { al }\end{array}$ & $\begin{array}{l}2016 \\
\text { Pubmed }\end{array}$ & $\begin{array}{l}\text { Prefrontal Cortex Activation Upon } \\
\text { a Demanding Virtual Hand- } \\
\text { Controlled Task: A New Frontier } \\
\text { for Neuroergonomics Revista: } \\
\text { Frontiers in Human Neuroscience }\end{array}$ & $\begin{array}{lr}\text { functional near-infrared } \\
\text { spectroscopy, } \\
\text { neuroergonomics, } \\
\text { controlled task, LEAP motion } \\
\text { controller, virtual reality, } \\
\text { remote control, brain activation }\end{array}$ \\
\hline $\begin{array}{l}\text { McKendrick } \\
\text { et al. }\end{array}$ & $\begin{array}{l}2014 \text { Web } \\
\text { of } \\
\text { Science }\end{array}$ & $\begin{array}{l}\text { Enhancing dual-task performance } \\
\text { with verbal and spatial working } \\
\text { memory training: Continuous } \\
\text { monitoring of cerebral } \\
\text { hemodynamics with NIRS } \\
\text { Revista: Neurolmage }\end{array}$ & $\begin{array}{l}\text { Working memory training, Near } \\
\text { infrared spectroscopy } \\
\text { Dorsolateral prefrontal cortex } \\
\text { Ventrolateral prefrontal cortex } \\
\text { Hemodynamics }\end{array}$ \\
\hline $\begin{array}{l}\text { Desoriere } \\
\text { et al. }\end{array}$ & $\begin{array}{l}2013 \\
\text { Scopus }\end{array}$ & $\begin{array}{l}\text { NIRS-measured pre frontal cortex } \\
\text { activity in neuroergonomics: } \\
\text { strengths and weaknesses } \\
\text { Revista: Frontiers in Human } \\
\text { Neuroscience }\end{array}$ & $\begin{array}{l}\text { mental workload, human- } \\
\text { computer interaction, cognitive } \\
\text { science, passive } \mathrm{BCl} \text {, attention }\end{array}$ \\
\hline Ayaz et al & $\begin{array}{l}2013 \\
\text { Pubmed }\end{array}$ & $\begin{array}{l}\text { Continuous monitoring of brain } \\
\text { dynamics with functional near } \\
\text { infrared spectroscopy as a tool } \\
\text { for neuroergonomic research: } \\
\text { empirical examples and a } \\
\text { technological development }\end{array}$ & $\begin{array}{l}\text { Human performance, fNIR, } \\
\text { Mental workload assessment, } \\
\text { Training } \\
\text { Air traffic control, Unmanned } \\
\text { aerial vehicle }\end{array}$ \\
\hline
\end{tabular}

Fonte: Dados da pesquisa.

Nesse caso o sangue oxigenado e desoxigenado pode ser contrastado por suas diferentes propriedades de absorção óptica, a fNIRS detecta os níveis desses parâmetros sanguíneos em resposta à atividade neuronal. Ao contrário de ressonância magnética e PET sua eficácia no mapeamento neurais vações dades entre regiões intimamente ligadas ou dentro de áreas corticais profundas é limitada devido à sua resolução espacial relativamente menor (METHA; PARASURAMAN, 2013).

Carriere et al (2016) afirmam que devido ao fato da fNIRS apresentar elevado grau de validade ecológica e sua exigência muito limitado de restrições físicas para indivíduos, poderia representar uma ferramenta válida para monitorar as respostas corticais no domínio da investigação da Neuroergonomia. 
Os autores acima citados concluíram em seu estudo que o fNIRS é uma aplicação promissora para avaliar objetivamente as alterações hemodinâmicas corticais que ocorrem em ambientes de realidade virtual. O desenvolvimento contínuo da tecnologia de fNIRS com o objetivo de entregar mais dispositivos dedicados, sofisticados e sem fio, juntamente com as mais avançadas soluções de realidade virtual, poderia fornecer a melhor abordagem combinada para monitorar formação de operadores e avaliar o trabalho mental. E sugere que estudos futuros poderão dar uma contribuição para uma melhor compreensão dos mecanismos cognitivos subjacentes desempenho humano quer no perito ou operadores não especializados.

Ayaz et al (2013) descrevem uma série de estudos sobre a avaliação de desempenho humano e aquisição de habilidades de monitoramento usando medidas de fNIRS da resposta hemodinâmica do córtex pré-frontal e sua relação com carga de trabalho mental, experiência e desempenho.

Os efeitos da carga de tarefas e conhecimentos sobre a resposta hemodinâmica pode ser confiável e sensível avaliada em uma série de tarefas, tarefas de laboratório padronizadas representativas de ambientes de trabalho real. No que diz respeito ao desenvolvimento de fNIRS portáteis sem fio, os resultados, embora preliminares, corroboram descobertas anteriores e apontam para o potencial do sistema fNIRS como um sensor usável, portátil e não invasivo para estudos futuros neuroergonômicos o que pode favorecer estudos futuros (AYAZ et al, 2013).

Para Desoriere et al (2013) e McKendrick et al (2014) a investigação da atividade cortical por fNIRS apresenta reais vantagens. Fornece uma maneira eficiente e eficaz para monitorar continuamente alterações hemodinâmicas durante longos períodos de tempo. Além disso, são sistemas portáteis estão sendo desenvolvidos como parte das iniciativas de imagem cerebral móvel a baixo custo.

Em consonância com os objetivos de neuroergonomia, essa tecnologia poderia ser usada para medir os efeitos do treinamento em tarefas complexas do mundo real onde o uso de fMRI seria um desafio ou impossível.

\subsection{Dopler Transcraniano (Transcranial Doppler Sonography - TCDS)}

Esta técnica usa ultrassom para captação da imagem do fluxo sanguíneo cerebral para os hemisférios cerebrais. TCDS utiliza um emissor de ultra-som aclopado à cabeça direcionado à artéria cerebral média (ACM) dentro do cérebro, e um receptor, em seguida, registra a freqüência da onda sonora refletida pelas células vermelhas do sangue que se deslocam através da artéria. $\mathrm{O}$ quadro3 mostra o artigo encontrado:

Quadro 3. Artigo selecionado - TCDS.

\begin{tabular}{|l|l|l|l|}
\hline Autores & Ano/Base & Título & Palavras-chaves \\
\hline \multirow{3}{*}{$\begin{array}{l}\text { Matthews } \\
\text { et al. }\end{array}$} & $\begin{array}{l}\text { The Psychometrics of Mental } \\
\text { Web of } \\
\text { Science }\end{array}$ & $\begin{array}{l}\text { Workload: Multiple Measures Are } \\
\text { Sensitive but Divergent } \\
\text { Revista: Human Factors }\end{array}$ & $\begin{array}{l}\text { performance, psychometrics, } \\
\text { psychophysiology, } \\
\text { neuroergonomics }\end{array}$ \\
\hline
\end{tabular}

\section{Fonte: Dados da Pesquisa.}

Em resposta ao aumento da atividade neuronal relacionado com a tarefa, sangue a velocidade fluxo aumenta para remover subprodutos da troca metabólica, que é capturado usando TCDS. TCDS tornou-se cada vez mais popular em estudos ergonômicos neuro cognitivas de vigilância e carga menta. No entanto, porque o volume de sangue cerebral e velocidade do fluxo sanguíneo é influenciada por alterações sistêmicas como a frequência cardíaca e da pressão arterial durante o exercício, TCDS é menos popular na avaliação da atividade neuronal relacionado com a tarefa em estudos neuroergonômicos físicos de fadiga (METHA;PARASURAMAN, 2013). 
O equipamento tem tamanho moderado, mas não possui uma resolução muito boa. $O$ exame permite a monitorização em tempo real das flutuações da velocidade de fluxo sanguíneo cerebral durante o repouso e durante a execução de algum paradigma de ativação cortica (HARATZ, 2014).

Matthews et al (2013) ao avaliarem diversos métodos de análise da carga de trabalho mental (eletroencefalograma, eletrocardiograma, espectrografia funcional do infravermelho próximo, dopller transcraniano e eye tracking) concluíram que todos mostram sensibilidade à carga de trabalho, e para a maioria, há fundamentado na literatura diagnosticidade e sensibilidade.

\subsection{Eletroencefalograma (Electroencephalography - EEG)}

O registro do EEG é realizado através das diferenças de tensão entre eletrodos ativos localizados em diferentes posições do couro cabeludo, como frontal, parietal, temporal e occipital de acordo com o Sistema Internacional 10-20 e um eletrodo de referência, geralmente colocado na orelha. O quadro 4 a seguir mostra a relação dos artigos encontrados.

Quadro 4. Artigos selecionados - EEG.

\begin{tabular}{|c|c|c|c|}
\hline Autores & Ano/Base & Título / Revista & Palavras-chaves \\
\hline $\begin{array}{l}\text { Giraudet } \\
\text { et al. }\end{array}$ & \multirow{4}{*}{$\begin{array}{l}2015 \\
\text { Web of } \\
\text { Science }\end{array}$} & $\begin{array}{l}\text { The neuroergonomic evaluation of human } \\
\text { machine interface design in air traffic } \\
\text { control using behavioral and EEG/ERP } \\
\text { measures } \\
\text { Revista: Behavioural Brain Research }\end{array}$ & $\begin{array}{l}\text { Air Traffic Control } \\
\text { Attentional resources ERP } \\
\text { Human machine interface } \\
\text { evaluation } \\
\text { Neuroergonomics }\end{array}$ \\
\hline $\begin{array}{l}\text { Brouwer } \\
\text { et al. }\end{array}$ & & $\begin{array}{l}\text { Using neurophysiological signals that } \\
\text { reflect cognitiveoraffective state: six } \\
\text { recommendations to avoid common pitfalls } \\
\text { Revista: Frontiers in Neuroscience }\end{array}$ & $\begin{array}{l}\text { passive } B C l, \text { physiological } \\
\text { computing, mental state } \\
\text { estimation, affective } \\
\text { computing, } \\
\text { neuroergonomics, EEG, } \\
\text { applied neuroscience }\end{array}$ \\
\hline $\begin{array}{l}\text { Yufeng } \\
\text { et al }\end{array}$ & & $\begin{array}{l}\text { Towards an effective cross-task mental } \\
\text { workload recognition model using } \\
\text { electroencephalography based on feature } \\
\text { selection and support vector machine } \\
\text { regression } \\
\begin{array}{l}\text { Revista: International Journal of } \\
\text { Psychophysiology }\end{array}\end{array}$ & $\begin{array}{l}\text { Mental workload, } \\
\text { Electroencephalography } \\
\text { Cross-task, Recursive } \\
\text { feature } \quad \text { elimination, } \\
\text { Support vector machine }\end{array}$ \\
\hline $\begin{array}{l}\text { Stasi } \\
\text { al }\end{array}$ & & $\begin{array}{l}\text { Task complexity modulates pilot } \\
\text { electroencephalographic activity during } \\
\text { real flights International Journal of } \\
\text { Revista: } \\
\text { Psychophysiology }\end{array}$ & $\begin{array}{l}\text { EEG, Fatigue, } \\
\text { Neuroergonomics, Safety, } \\
\text { Simulation, Training }\end{array}$ \\
\hline
\end{tabular}

Fonte: Dados da Pesquisa.

Os sinais do EEG apresentam excelente resolução temporal pois as bandas de frequências diferentes, são associadas a vários estados físicos e cognitivos. Análise espectral desses sinais pode ser realizada para avaliar a potência em diferentes faixas de frequência: delta (0,5-3 Hz), theta (4-8 Hz), alfa (8-13 Hz), beta (13-30 Hz), e gama (40-50 Hz) (METHA; PARASURAMAN, 2013). Segundo esses autores a EEG tem baixo custo de utilização.

Yufeng et al (2015) sugerem um modelo baseado em EEG capaz de discriminar carga de trabalho para qualquer tarefa e afirma que o EEG é uma medida psicofisiológica potencial da carga mental. Stasi et al (2015) utilizou o EEG na avaliação do desempenho cognitivo de pilotos durante vôos reais, sugerindo esta técnica na comprenção da atividade cerebral e na performance cognitiva desses profissionais durante suas atividades e em diferentes dificuldades. 
Análises baseadas na estimativa estado mental através de sinais neurofisiológicos promovem melhora no desempenho ou no bem-estar dos usuários, além do uso de uma comparação sensata aplicação. O campo de estimação de estado mental através de sinais neurofisiológicos como uma ciência irá beneficiar de um comportamento cuidadoso dos cientistas como a declarações de que é possível e potencialmente útil e o que não, bem como de estudos de alta qualidade que evitar erros comuns nesse tipo de estudo (BROUWER et al, 2015).

Giraudet et al (2015) usando abordagem neuroergonômica para análise da carga cognitiva em tarefa simulada de Controle de Tráfego Aéreo utilizou para tanto a amplitude da onda P300 obtida através de eletroencefalograma, conclue que a abordagem neuroergonomic favorece uma compreensão mais completa do complexo impacto das alterações no design de interface. Foi possível também confirmar que a amplitude do P300 representa um índice de carga cognitiva confiável em ambientes ecológicos.

\subsection{Magnetoencefalografia (Magnetoencephalography - MEG)}

Segundo Braeutigam (2013) magnetoencefalografia (MEG) baseia-se na detecção de campos magnéticos gerados pelas correntes que fluem nos neurônios. Ao contrário da tomografia por emissão de pósitrons (PET) ou ressonância magnética funcional (fMRI), MEG não depende de efeitos secundários induzidos pela atividade cerebral, mas mede diretamente os campos magnéticos gerados principalmente por correntes iônicas neuronais pós-sinápticas.

Em contraste com seu primo mais próximo, o eletroencefalograma (EEG), os sinais de MEG estão e essencialmente afetados por diferenças de condutividade sobre o fluxo magnético, proporcionando uma visão quase sem distorções de atividade cerebral, e simplificando a análise de dados e interpretação de padrões. MEG é principalmente sensível aos campos magnéticos gerados no córtex cerebral, mas, sistemas modernos de toda a cabeça com múltiplas configurações de sensor podem detectar a atividade em regiões subcorticais (BRAEUTIGAM, 2013).

\subsection{Potenciais Relacionados a Eventos (Event-related Potentials - ERP's)}

Os ERP'são derivados da eletroencefalografia e representam a resposta neural do cérebro para eventos sensoriais, motores e cognitivos específicos. Representam o resultado da média dos sinais de EEG em períodos de tempo fechados em resposta a um estímulo ou evento particular (METHA; PARASURAMAN, 2013). Foram encontrados os seguintes trabalhos:

Quadro 5. Artigos selecionados- ERP.

\begin{tabular}{|l|l|l|l|}
\hline Autores & Ano/Base & Título & Palavras-chaves \\
\hline $\begin{array}{l}\text { Roy et } \\
\text { al. }\end{array}$ & $\begin{array}{l}2015 \\
\text { Scopus }\end{array}$ & $\begin{array}{l}\text { A Comparison of ERP Spatial Filtering for Optimal Mental Workload } \\
\text { Estimation } \\
\text { Revista: IEEE }\end{array}$ & $\begin{array}{l}\text { Neuroergonomics } \\
\text { ERP }\end{array}$ \\
\hline $\begin{array}{l}\text { Takeda } \\
\text { et al. }\end{array}$ & $\begin{array}{l}2014 \mathrm{Web} \\
\text { of } \\
\text { Science }\end{array}$ & $\begin{array}{l}\text { Electrophysiological measurement of } \\
\text { interest during walking in a simulated } \\
\text { environment } \\
\text { Revista: International Journal of } \\
\text { Psychophysiology }\end{array}$ & $\begin{array}{l}\text { Task-irrelevant probe } \\
\text { Event-related potentials } \\
\text { Eye-fixation-related potentials } \\
\text { Simulator }\end{array}$ \\
\hline
\end{tabular}

Fonte: Dados da pesquisa.

Normalmente o equipamento tem baixo custo. Para avaliar a carga de trabalho mental ou o erro humano, formas de onda dos ERP's são examinados quanto a mudanças na amplitude e latência de diferentes componentes, geralmnete definidos como atividade de picopositivo ou negativo (como os componentes P3 e N1), assim como, quanto a atividade subindo 
lentamente, por exemplo, potencial de prontidão lateralizado, o que tras positivos ganhos de resolução temporal (LUCK, 2005 apud METHA; PARASURAMAN, 2013).

Para Roy et al (2015) e Takeda et al (2014) a estimativa da carga de trabalho mental é de interesse crucial para interfaces de utilizador adaptáveis e neuroergonomia e podem ser uma técnica poderosa e útil para estimar estado mental. Ainda de acordo com o estudo acima citado, os autores mostram que as que a estimativa da carga de trabalho usando ERP's é mais do que viável e deve ser considerado para implementação do sistema de monitoramento do estado mental.

3.9 Estimulação Elétrica Transcraniana (Transcranial Direct Current Stimulation - tCDS) e Estimulação Magnética Transcraniana (Transcranial Magnetic Stimulation - TMS)

A Estimulação Elétrica Transcraniana é um dispositivo neuromodulador frequentemente publicitada por sua capacidade de melhorar o desempenho cognitivo e comportamental (HORVATH; CARTER; FORTE, 2014).

A Estimulação Magnética Transcraniana é uma técnica não invasiva que utiliza a curta e rápida mudança pulsos de campo magnético para induzir correntes elétricas no tecido cortical subjacente. Através da aplicação de impulsos repetidos (estimulação magnética transcraniana repetitiva) a baixas frequências (por exemplo, $1 \mathrm{~Hz}$ ), pode-se suprimir a atividade cortical subjacente e de alta frequência a estimulação (e.g., $20 \mathrm{~Hz}$ ) podem resultar em alterações excitatórios (FOX et al, 2012)

Estas técnicas permitem a inibição temporária ou activação de regiões cerebrais específicas permitindo assim aos investigadores examinar o papel causal de diferentes regiões do cérebro em várias funções cognitivas. Ambas as técnicas também podem ser utilizadas para modular a actividade cerebral de modo a que o desempenho de uma dada tarefa cognitiva ou motor é melhorada (METHA;PARASURAMAN, 2013). Segundos os autores supra citados ambos métodos possuem baixo custos, e a diferença está na postabilidade do equipamento que a TMS praticamente é estático e o tDCS móvel.

\subsection{Temas Relacionados com os Métodos Apresentados}

Outros artigos foram encontrados que não falam diretamente de um ou outro método, entretanto exploram algum tema de conexão. Por exemplo, Stasi el al (2013a) avaliaram a correlação da velocidade sacádica com parâmetros da tarefa em clínica, militar e situações cotidianas, e seu potencial papel na ergonomia. E concluiram que as medidas sacádicas variam de acordo com a dificuldade da tarefa e com o time-on-task em cenários naturalistas. Foram explorados achados históricos e recentes e discutida hipótese de que mudanças na velocidade sacádica indicam variações na ativação do sistema nervoso simpático; isto é, variações de excitação e esse fato pode auxiliar na escolha do método.

A seguir o quadro com artigos encontrados com assuntos paralelos:

Quadro 6. Artigos selecionados - Assuntos paralelos norteadores do método

\begin{tabular}{|c|c|c|c|}
\hline Autores & Ano/Base & Título & Palavras-chaves \\
\hline \multirow{3}{*}{ Stati et $A L$} & $\begin{array}{l}2013 \\
\text { Science } \\
\text { Direct }\end{array}$ & $\begin{array}{l}\text { Saccadic velocity as an arousal } \\
\text { index in naturalistic tasks } \\
\text { Revista: Neuroscience and } \\
\text { Biobehavioral Reviews }\end{array}$ & $\begin{array}{l}\text { Arousal neurons; Omnipause } \\
\text { neurons } \\
\text { Depressive disorder; Mental } \\
\text { workload; Mental fatigue; } \\
\text { Cognitive load; Main sequence }\end{array}$ \\
\hline & $\begin{array}{l}2011 \\
\text { Science } \\
\text { Direct }\end{array}$ & $\begin{array}{l}\text { A neuroergonomic approach to } \\
\text { evaluating mental workload in } \\
\text { hypermedia Interactions - } \\
\text { Revista: International Journal of } \\
\text { Industrial Ergonomics }\end{array}$ & $\begin{array}{l}\text { Eye movements, Saccadic } \\
\text { peak velocity, Hypermedia, E- } \\
\text { commerce, } \\
\text { Main sequenc, Pupil, Events- } \\
\text { interface }\end{array}$ \\
\hline & 2013 Web & Microsaccade and drift dynamics & air traffic control, fixation, \\
\hline
\end{tabular}




\begin{tabular}{|c|c|c|c|}
\hline & $\begin{array}{l}\text { of } \\
\text { Science }\end{array}$ & $\begin{array}{llr}\text { reflect mental fatigue - Revista: } \\
\text { European } & \text { Journal } & \text { of } \\
\text { Neuroscience } & & \end{array}$ & $\begin{array}{l}\text { fixational eye movements, free- } \\
\text { viewing, main sequence, } \\
\text { neuroergonomics. }\end{array}$ \\
\hline Parasuraman & $\begin{array}{l}2011 \\
\text { Scopus }\end{array}$ & $\begin{array}{l}\text { Neuroergonomics: Brain, } \\
\text { Cognition, and Performance at } \\
\text { Work } \\
\text { Revista: Current Directions in } \\
\text { Psychological Science }\end{array}$ & $\begin{array}{lr}\text { neuroergonomics, } & \text { molecular } \\
\text { genetics, multitasking, mental } \\
\text { workload, error, adaptive } \\
\text { interfaces, } & \text { individual } \\
\text { differences } & \\
\end{array}$ \\
\hline $\begin{array}{l}\text { Metha; } \\
\text { Parasuraman }\end{array}$ & $\begin{array}{l}2013 \\
\text { Scopus }\end{array}$ & $\begin{array}{l}\text { Neuroergonomics: a review of } \\
\text { applications to physical and } \\
\text { cognitive work - Revista: Frontiers } \\
\text { in Human Neuroscience }\end{array}$ & $\begin{array}{l}\text { physical work parameters, } \\
\text { physical fatigue, mental } \\
\text { fatigue, vigilance, training, } \\
\text { neuroadaptive systems }\end{array}$ \\
\hline
\end{tabular}

Fonte: Dados da Pesquisa.

Nessa direção, em tempos de tecnologia da informação, as mudanças na carga mental de trabalho poderia ter impactos significativos sobre o desempenho do operador, possivelmente causando atrasos no processamento de informações ou até mesmo causar operadores para ignorar as informações de entrada. Nesse sentido, a pesquisa neuroergonômica identificou a necessidade de acompanhar os estados em tempo real do operador, a fim de reduzir e prevenir erros e determinar o tipo eo nível de assistência automatizada é mais adequada para ajudar os operadores para completar tarefas (STASI et al, 2011).

Segundo Stasi et al (2013b) a fadiga mental pode modular a dinâmica do movimentos sacádicos. Em seu estudo verificou que a velocidade sacádica e microsacádica diminuiu com o tempo na tarefa, enquanto velocidade de deriva aumentou, sugerindo que a instabilidade ocular aumenta com a fadiga mental. Propoem que as variações na dinâmica de movimento dos olhos com o tempo na tarefa são consistentes com a ativação do centro de sono do cérebro em correlação com a fadiga mental. Concluíram que alterações na dinâmica desses movimentos pode indicar fadiga mental devido ao tempo na tarefa, independentemente da complexidade da tarefa.

Já Parasuraman (2011) afirma que os estudos em neuroergonomia ilustram o valor, bem como os limites da abordagem neuroergonômica. Sendo que esta não substitui métodos comportamentais convencionais de análise, no entanto, fornece uma ferramenta adicional que tem sido útil tanto em teorias de refino da carga de trabalho mental quanto no fornecimento de técnicas alternativas para a sua avaliação em ambientes de trabalho.

\subsection{Comparação entre métodos}

Com base nos artigos apresentados e principalmente em Metha e Parasuraman (2013) é possivel comparar os métodos neuroergonômicos com base em três critérios: 1 . Portabilidade; 2. Custos; 3. Resolução Espacial e 4. Resolução temporal, ao que se segue.

Quadro 7. Lista das técnicas neuroergonômicas e suas características

\begin{tabular}{|l|l|l|l|l|}
\hline Método & $\begin{array}{l}\text { Portabilidade/ } \\
\text { Mobilidade }\end{array}$ & Custo & $\begin{array}{l}\text { Resolução } \\
\text { Espacial }\end{array}$ & $\begin{array}{l}\text { Resolução } \\
\text { Temporal }\end{array}$ \\
\hline MRI & Nenhuma & Alto & Alta & Não Avalia \\
\hline DTI & Nenhuma & Alto & Alta & Não Avalia \\
\hline fMRI/PET & Nenhuma & Alto & Alta & Baixa \\
\hline fNIRS & Alta & Baixa & Moderada & Baixa \\
\hline TCDS & Moderada & Baixa & Baixa & Baixa \\
\hline EEG & Moderada & Baixa & Baixa & Alta \\
\hline ERP & Moderada & Baixa & Baixa & Alta \\
\hline TMS & Baixa & Moderado & Alta & Alta \\
\hline tDCS & Alta & Muito Baixo & Baixa & Baixa \\
\hline
\end{tabular}

Fonte: dos autores a partir de Metha e Parasuraman (2013). 


\section{CONSIDERAÇÕES FINAIS}

A carga mental de trabalho é definida, de maneira mais ampla, como a interação entre as exigências da tarefa e a capacidade do indivíduo de realizá-la, sendo sua avaliação uma medida quanti ou qualitativa dos níveis de atividade neurofisiológica da carga mental para realização da tarefa. O estudo da mesma continua a ser um importante tema para a pesquisa e prática ergonômica.

A neuroergonomia trata-se de uma vertente da ergonomia cognitiva que busca, com recursos de neuroimagem e neurociências, entender os mecanismos neurais relacionados à tarefa/trabalho. Estudos recentes vêm demonstrando a importância da realização de pesquisas em ambientes naturais, ou seja, no próprio ambiente de trabalho. Com isso, observariam-se resultados mais reais, representativos do indivíduo em ação.

Diante do exposto, e ainda sabendo que a carga mental de trabalho envolve mecanismos mentais de tomada de decisão e tratamento da informação, e que este gerenciamento é realizado por nossas estruturas neurais superiores, podemos considerar os métodos de análise neuroergonômica como alternativas para sua análise.

Como abordado mais acima, os estudos hoje procuram aproximar-se o máximo possível do ambiente real de trabalho, avaliando situações reais e dinâmicas que só podem ser observadas nesses ambientes. Para tanto, os métodos que oferecem maior mobilidade são os mais adequados, como o EEG e a fNIRS, que a cada dia, com o avanço tecnológico tornam-se mais portáteis e ganham versões que cada vez mais favorecem seu uso nos mais variados ambientes e situações e isso fica evidente no grande número de estudos que escolheram estas técnicas para a análise neuroergonômica.

\section{REFERÊNCIAS BIBLIOGRÁFICAS}

ANTONELLI et al. Avaliação da carga de trabalho físico em trabalhadores de uma fundição através da variação da frequência cardíaca e análise ergonômica do trabalho. Ação Ergonômica, volume 6, número 2, 2011.

AYAZ et al. Optical brain monitoring for operator training and mental workload assessment. Neurolmage, Volume 59, 2012.

BENEDETO et al. Driver workload and eye blink duration. Transportation Research Part F, volume 14, p. 199-208, 2011.

BRAEUTIGAM, S. Magnetoencephalography: Fundamentals and Established and Emerging Clinical Applications in Radiology. Hindawi Publishing Corporation ISRN Radiology, http://dx.doi.org/10.5402/2013/529463, 2013.

BROUWER et al. Using neurophysiological signals that reflect cognitive or affective state: six recommendations to avoid common pitfalls. Frontiers in Neuroscience, Volume 9, Article 136. DOI: 10.3389/fnins.2015.00136, 2015.

CARRIERE et al. Prefrontal Cortex Activation Upon a Demanding Virtual HandControlled Task: A New Frontier for Neuroergonomics. Frontiers in Human Neuroscience, Volume 10, Article 53. DOI: 10.3389/fnhum.2016.00053, 2016.

DERORIĖRE et al. NIRS - measured prefrontal cortex activity in neuroergonomics: strengths and weaknesses. Frontiers in Human Neuroscience. Volume 7, Article 583. DOI: 10.3389/fnhum.2013.00583, 2013.

FOX et al. Efficacy of Transcranial Magnetic Stimulation Targets for Depression Is Related to Intrinsic Functional Connectivity with the Subgenual Cingulate. Biol Psychiatr, Volume 72, p. 595-603, 2012 
GIRAUDET et al. The neuroergonomic evaluation of human machine interface design in air traffic control using behavioral and EEG/ERP measures. Behavioural Brain Research, Volume 294, p. 246-253, 2015.

HARATZ, S. S. Nova metodologia de Doppler transcraniano funcional durante tarefa motora unimanual. 2014. 133 f. Tese de Doutorado (Doutorado em Ciências). Faculdade de Medicina da Universidade de São Paulo, Universidade de São Paulo, São Paulo.

HORVATH, J. C.; CARTER, O.; FORTE J. D. Transcranial direct current stimulation: five important issues we aren't discussing (but probably should be). Frontiers in Systems Neuroscience, Volume 8, 2014.

INTERNATIONAL ERGONOMICS ASSOCIATION. Definição Internacional de Ergonomia. Revista Ação Ergonômica. Volume 3, Número 2, set/2008 ISSN 1519-7859.

KRAMER, A. F.; PARASURAMAN, R. Neuroergonomics: Applications of Neuroscience to Human Factors. In: CACIOPPO, J. T.; TASSINARY, L. G.; BERNTSON, G. G. Handbook of Psychophysiology. Reino Unido: Cambridge University Press, 2007.

MATTHEWS et al. The Psychometrics of Mental Workload: Multiple Measures Are Sensitive but Divergent. Human Factors, Volume 57, Número 1, p. 125 -143, DOI: 10.1177/0018720814539505, 2015.

MÁSCULO, F.S.; VIDAL, M.C. Introdução à Ergonomia. In: MÁSCULO, F.S.; VIDAL, M.C. Ergonomia: Trabalho adequado e eficiente. Rio de Janeiro: Elsevier, 2011.

METHA, R.K; PARASURAMAN, R. Neuroergonomics: a review of applications to physical and cognitive work. Frontiers in Human Neuroscience., Volume 7, Article 889, 2013.

MOTTER, A. A.; SANTOS, M.; GUIMARÃES, A. T. B. O que está à sombra na carga de trabalho de estivadores?. Revista Produção Online, Florianópolis, SC, v.15, n. 1, p. 321344, jan./mar. 2015.

PARASURAMAN, R. Neuroergonomics: Brain, Cognition, and Performance at Work. Current Directions in Psychological Science, Volume 20, Número 3, p. 181-186, DOI: 10.1177/0963721411409176, 2011.

PARASURAMAN, R.; CHRISTENSEN, J. GRAFTON, S. Neuroergonomics: The brain in action and at work. Neurolmage, Volume 59, p. 1-3, 2012.

POLDRACK, R.; MUMFORD, J. A.; NICHOLS, T. E. Handbook of Functional MRI Analysis. New York: Cambridge University Press, 2011. DOI: 10.1017/CBO9780511895029.

ROY et al. A Comparison of ERP Spatial Filtering Methods for Optimal Mental Workload Estimation. IEEE, DOI: 978-1-4244-9270-/15, 2015.

STASI et al. A neuroergonomic approach to evaluating mental workload in hypermedia interactions. International Journal of Industrial Ergonomics, Volume 41, p. 298-304, 2011.

STASI et al (a). Saccadic velocity as an arousal index in naturalistic tasks. Neuroscience and Biobehavioral Reviews, Volume 37, p. 968-975, 2013.

STASI et al (b). Microsaccade and drift dynamics reflect mental fatigue. European Journal of Neuroscience, Volume 38, p. 2389-2398, 2013.

STASI et al. Task complexity modulates pilot electroencephalographic activity during real flights. Psychophysiology, Volume 52, p. 951-956, 2015.

TAKEDA et al. Electrophysiological measurement of interest during walking in a simulated environment. International Journal of Psychophysiology, Volume 93, p. 363-370, 2014. 
UEHARA, E.; MORAES, A. Neuroergonomia: um novo campo na ergonomia do século XXI?. In: Anais do $11^{\circ}$ Congresso Internacional de Ergonomia e Usabilidade de Interface Humano-Tecnologia: Produto, Informações, Ambiente construído e Transporte ERGODESING USICH, Manaus, 2011.

YUFENG et al. Towards an effective cross-task mental workload recognition model using electroencephalography based on feature selection and support vector machine regression. International Journal of Psychophysiology, Volume 98, p. 157-166, 2015. 